\title{
Solvability and Optimal Controls of Semilinear Riemann-Liouville Fractional Differential Equations
}

\author{
Xue Pan, Xiuwen Li, and Jing Zhao \\ College of Sciences, Guangxi University for Nationalities, Nanning, Guangxi 530006, China \\ Correspondence should be addressed to Xue Pan; xuepan100@126.com
}

Received 4 January 2014; Accepted 10 March 2014; Published 15 April 2014

Academic Editor: Simeon Reich

Copyright (C) 2014 Xue Pan et al. This is an open access article distributed under the Creative Commons Attribution License, which permits unrestricted use, distribution, and reproduction in any medium, provided the original work is properly cited.

\begin{abstract}
We consider the control systems governed by semilinear differential equations with Riemann-Liouville fractional derivatives in Banach spaces. Firstly, by applying fixed point strategy, some suitable conditions are established to guarantee the existence and uniqueness of mild solutions for a broad class of fractional infinite dimensional control systems. Then, by using generally mild conditions of cost functional, we extend the existence result of optimal controls to the Riemann-Liouville fractional control systems. Finally, a concrete application is given to illustrate the effectiveness of our main results.
\end{abstract}

\section{Introduction}

The purpose of this paper is to investigate the solvability and optimal controls for the following semilinear control systems with Riemann-Liouville fractional derivatives:

$$
\begin{gathered}
{ }^{L} D_{t}^{\alpha} x(t)=A x(t)+B u(t)+f(t, x(t)), \\
t \in(0, b], \quad u \in U_{\mathrm{ad}}, \\
\left.I_{0^{+}}^{1-\alpha} x(t)\right|_{t=0}=x_{0} \in X,
\end{gathered}
$$

where $0<\alpha \leq 1,{ }^{L} D_{t}^{\alpha}$ denotes the Riemann-Liouville fractional derivative of order $\alpha$ with the lower limit zero. $A: D(A) \subseteq X \rightarrow X$ is the infinitesimal generator of a $C_{0}$-semigroup $T(t)(t \geq 0)$ on a separable Banach space $X$. $f:[0, b] \times X \rightarrow X$ is a given function to be specified later. The control function $u$ is given in a suitable admissible control set $U_{\mathrm{ad}} . B$ is a linear operator from a separable reflexive Banach space $Y$ into $X$. The cost functional over the family of admissible state control pair $(x, u)$ is given by

$$
\mathscr{J}(x, u)=\int_{J} \mathscr{L}(t, x(t), u(t)) d t .
$$

In recent years, fractional calculus has been paid more and more attention that lies on the fact that it allows us to consider integration and differentiation of any order, not necessarily integer, and fractional-order models are more accurate than integer-order models; that is, there are more degrees of freedom in the fractional-order models. Furthermore, fractional derivatives provide an excellent instrument for the description of memory and hereditary properties of various materials and processes due to the existence of term which insures the history and its impact on the present and future in a model. Therefore, it has drawn great applications of the mathematical modeling of systems and processes in the fields of physics, chemistry, aerodynamics, electrodynamics of complex medium, viscoelasticity, heat conduction, electricity mechanics, control theory, and so forth. For more details on these topics one can see, for instance, [1-17] and the reference therein.

The definitions of Riemann-Liouville fractional derivatives or integrals initial conditions play an important role in some practical problems. Heymans and Podlubny [18] have demonstrated that it is possible to attribute physical meaning to initial conditions expressed in terms of RiemannLiouville fractional derivatives or integrals on the field of the viscoelasticity, and such initial conditions are more appropriate than physically interpretable initial conditions.

Since the pioneering work on the fundamental solutions of Caputo fractional evolution equations associated with some probability densities has been reported by El-Borai $[19,20]$, the study of the existence, controllability, and optimal controls of the fractional semilinear functional differential 
equations and inclusions with the infinitesimal generator $A$ of a $C_{0}$-semigroup have been extensively studied (one can see [21-23]). However, to the best of our knowledge, the solvability and optimal controls for fractional semilinear differential equations with Riemann-Liouville fractional derivatives are still untreated topics in the literature and this fact is the motivation of the present work. Our aim in this paper is to provide some suitable sufficient conditions for the existence and uniqueness of solutions and optimal control results corresponding to the admissible control sets of fractional abstract Cauchy problems with the RiemannLiouville fractional derivatives.

The rest of this paper is organized as follows. In Section 2, we will present some basic definitions and preliminary facts, such as definitions, lemmas, and theorems, which will be used throughout the following sections. In Section 3, by applying the well-known fixed point theorem, some sufficient conditions are established for the existence and uniqueness of mild solutions of the system (1). In Section 4, we will study the optimal controls for semilinear differential equations with Riemann-Liouville fractional derivatives. Finally, we present an example to demonstrate our main results in Section 5.

\section{Preliminaries}

In this section, we introduce some basic definitions and preliminaries which are used throughout this paper. The norm of a Banach space $X$ will be denoted by $\|\cdot\|_{X} \cdot L_{b}(X, Y)$ denotes the space of bounded linear operators from $X$ to $Y$. For the uniformly bounded $C_{0}$-semigroup $T(t)$, we set $M:=\sup _{t \geq 0}\|T(t)\|_{L_{b}(X)}<\infty$. Let $C(J, X)$ denote the Banach space of all $X$-value continuous functions from $J=[0, b]$ into $X$ with the norm $\|x\|_{C}=\sup _{t \in J}\|x(t)\|_{X}$. Let $A C(J, X)$ be the space of functions $f$ which are absolutely continuous on $J$ and $A C^{m}(J, X)=\left\{f: J \rightarrow X\right.$ and $\left.f^{(m-1)}(x) \in A C(J, X)\right\}$. To define the mild solutions of (1), we consider the Banach space $C_{1-\alpha}(J, X)=\left\{x: t^{1-\alpha} x(t) \in C(J, X)\right\}$ with the norm $\|x\|_{C_{1-\alpha}}=\sup \left\{t^{1-\alpha}\|x(t)\|_{X}: t \in J\right\}$. Obviously, the space $C_{1-\alpha}(J, X)$ is a Banach space.

Firstly, let us recall the following definitions from fractional calculus. For more details, one can see $[3,16]$.

Definition 1. The integral

$$
I_{t}^{\alpha} f(t)=\frac{1}{\Gamma(\alpha)} \int_{0}^{t}(t-s)^{\alpha-1} f(s) d s, \quad \alpha>0,
$$

is called Riemann-Liouville fractional integral of order $\alpha$, where $\Gamma$ is the gamma function.

Definition 2. For a function $f(t)$ given in the interval $[0, \infty)$, the expression

$$
{ }^{L} D_{t}^{\alpha} f(t)=\frac{1}{\Gamma(n-\alpha)}\left(\frac{d}{d t}\right)^{n} \int_{0}^{t}(t-s)^{n-\alpha-1} f(s) d t
$$

where $n=[\alpha]+1,[\alpha]$ denotes the integer part of number $\alpha$ and is called the Riemann-Liouville fractional derivative of order $\alpha$.
Lemma 3 (see [3]). Let $\alpha>0$, let $m=[\alpha]+1$, and let $x_{m-\alpha}(t)=I_{0^{+}}^{m-\alpha} x(t)$ be the fractional integral of order $m-\alpha$. If $x(t) \in L^{1}(J, X)$ and $x_{m-\alpha}(t) \in A C^{m}(J, X)$, then one has the following equality:

$$
I_{t}^{\alpha L} D_{t}^{\alpha} x(t)=x(t)-\sum_{k=1}^{m} \frac{x_{m-\alpha}^{(m-k)}(0)}{\Gamma(\alpha-k+1)} t^{\alpha-k} .
$$

The Laplace transform formula for the Riemann-Liouville fractional integral is defined by

$$
L\left\{I_{t}^{\alpha} u(t) ; \lambda\right\}=\frac{1}{\lambda^{\alpha}} \widehat{u}(\lambda)
$$

where $\widehat{u}(\lambda)$ is the Laplace of $u$ defined by

$$
\begin{array}{r}
\widehat{u}(\lambda)=\int_{0}^{\infty} e^{-\lambda t} u(t) d t, \quad \operatorname{Re} \lambda>\omega, \\
|u(t)| \leq c e^{\omega t}, \quad c \text { is a constant. }
\end{array}
$$

Lemma 4. Let $\alpha \in(0,1]$ and $h \in L^{p}(J, X), p>1 / \alpha$, if $x(t) \in L^{1}(J, X), x_{1-\alpha}(t) \in A C(J, X)$, and $x$ is a solution of the following problem:

$$
\begin{gathered}
{ }^{L} D_{t}^{\alpha} x(t)=A x(t)+h(t), \quad t \in(0, b], \\
\left.I_{0^{+}}^{1-\alpha} x(t)\right|_{t=0}=x_{0} \in X
\end{gathered}
$$

then, $x$ satisfies the following equation:

$$
x(t)=t^{\alpha-1} T_{\alpha}(t) x_{0}+\int_{0}^{t}(t-s)^{\alpha-1} T_{\alpha}(t-s) h(s) d s, \quad t \in J,
$$

where

$$
\begin{gathered}
T_{\alpha}(t)=\alpha \int_{0}^{\infty} \theta \xi_{\alpha}(\theta) T\left(t^{\alpha} \theta\right) d \theta, \\
\xi_{\alpha}(\theta)=\frac{1}{\alpha} \theta^{-1-(1 / \alpha)} \omega_{\alpha}\left(\theta^{-(1 / \alpha)}\right), \\
\omega_{\alpha}(\theta)=\frac{1}{\pi} \sum_{n=1}^{\infty}(-1)^{n-1} \theta^{-n \alpha-1} \frac{\Gamma(n \alpha+1)}{n !} \sin (n \pi \alpha), \\
\theta \in(0, \infty) ;
\end{gathered}
$$

and $\xi_{\alpha}$ is a probability density function defined on $(0, \infty)$, that is,

$$
\xi_{\alpha}(\theta) \geq 0, \theta \in(0, \infty), \quad \int_{0}^{\infty} \xi_{\alpha}(\theta) d \theta=1 .
$$

Proof. Apply Riemann-Liouville fractional integral operator on both sides of (8); then, by Lemma 3, we get

$$
\begin{aligned}
x(t) & =\frac{\left.I_{0^{+}}^{1-\alpha} x(t)\right|_{t=0}}{\Gamma(\alpha)} t^{\alpha-1}+I_{t}^{\alpha} A x(t)+I_{t}^{\alpha} h(t) \\
& =\frac{t^{\alpha-1}}{\Gamma(\alpha)} x_{0}+I_{t}^{\alpha} A x(t)+I_{t}^{\alpha} h(t)
\end{aligned}
$$

that is,

$$
x(t)=\frac{t^{\alpha-1}}{\Gamma(\alpha)} x_{0}+\frac{1}{\Gamma(\alpha)} \int_{0}^{t}(t-s)^{\alpha-1}[A x(s)+h(s)] d s .
$$


Let $\lambda>0$; taking the Laplace transformations

$$
\widehat{x}(\lambda)=\int_{0}^{\infty} e^{-\lambda t} x(t) d t, \quad \widehat{h}(\lambda)=\int_{0}^{\infty} e^{-\lambda t} h(t) d t,
$$

to (13), we obtain

$$
\begin{aligned}
\widehat{x}(\lambda) & =\frac{1}{\lambda^{\alpha}} x_{0}+\frac{1}{\lambda^{\alpha}} A \widehat{x}(\lambda)+\frac{1}{\lambda^{\alpha}} \widehat{h}(\lambda) \\
& =\left(\lambda^{\alpha} I-A\right)^{-1} x_{0}+\left(\lambda^{\alpha} I-A\right)^{-1} \widehat{h}(\lambda) \\
& =\int_{0}^{\infty} e^{-\lambda^{\alpha} t} T(t) x_{0} d t+\int_{0}^{\infty} e^{-\lambda^{\alpha} t} T(t) \widehat{h}(\lambda) d t .
\end{aligned}
$$

Consider the one-sided stable probability density

$$
\begin{array}{r}
\varpi_{\alpha}(\theta)=\frac{1}{\pi} \sum_{n=1}^{\infty}(-1)^{n-1} \theta^{-n \alpha-1} \frac{\Gamma(n \alpha+1)}{n !} \sin (n \pi \alpha), \\
\theta \in(0, \infty),
\end{array}
$$

whose Laplace transform is given by

$$
\int_{0}^{\infty} e^{-\lambda \theta} \varpi_{\alpha}(\theta) d \theta=e^{-\lambda^{\alpha}}, \quad \alpha \in(0,1) .
$$

Hence, it follows from (15) and (17) that

$$
\begin{aligned}
& \int_{0}^{\infty} e^{-\lambda^{\alpha} t} T(t) x_{0} d t \\
& =\int_{0}^{\infty} e^{-(\lambda s)^{\alpha}} T\left(s^{\alpha}\right) x_{0} d s^{\alpha} \quad\left(t=s^{\alpha}\right) \\
& =\alpha \int_{0}^{\infty} e^{-(\lambda s)^{\alpha}} T\left(s^{\alpha}\right) s^{\alpha-1} x_{0} d s \\
& =\alpha \iint_{0}^{\infty} \omega_{\alpha}(\theta) e^{-\lambda s \theta} T\left(s^{\alpha}\right) s^{\alpha-1} x_{0} d \theta d s \\
& =\alpha \iint_{0}^{\infty} \omega_{\alpha}(\theta) e^{-\lambda u} T\left(\frac{u^{\alpha}}{\theta^{\alpha}}\right) \frac{s^{\alpha-1}}{\theta^{\alpha}} x_{0} d u d \theta \quad(u=\theta s) \\
& =\int_{0}^{\infty} e^{-\lambda u}\left[\alpha \int_{0}^{\infty} \omega_{\alpha}(\theta) T\left(\frac{u^{\alpha}}{\theta^{\alpha}}\right) \frac{s^{\alpha-1}}{\theta^{\alpha}} x_{0} d \theta\right] d u, \\
& \int_{0}^{\infty} e^{-\lambda^{\alpha} t} T(t) \hat{h}(\lambda) d t \\
& =\int_{0}^{\infty} e^{-\lambda^{\alpha} t} T(t)\left[\int_{0}^{\infty} e^{-\lambda s} h(s) d s\right] d t \\
& =\iint_{0}^{\infty} e^{-\lambda^{\alpha} t} T(t) e^{-\lambda s} h(s) d s d t \\
& =\iint_{0}^{\infty} e^{-(\lambda \mu)^{\alpha}} T\left(\mu^{\alpha}\right) q \mu^{\alpha-1} e^{-\lambda s} h(s) d s d \mu \\
& \quad\left(t=\mu^{\alpha}\right)
\end{aligned}
$$

$$
\begin{aligned}
& =\iiint_{0}^{\infty} \alpha \omega_{\alpha}(\theta) e^{-\lambda \mu \theta} T\left(\mu^{\alpha}\right) \mu^{\alpha-1} e^{-\lambda s} h(s) d \theta d s d \mu \\
& =\iiint_{0}^{\infty} \alpha \omega_{\alpha}(\theta) e^{-\lambda v} T\left(\frac{\nu^{\alpha}}{\theta^{\alpha}}\right) \frac{\nu^{\alpha-1}}{\theta^{\alpha}} e^{-\lambda s} h(s) d \nu d \theta d s
\end{aligned}
$$$$
(\nu=\mu \theta)
$$

$$
\begin{aligned}
& =\iiint_{0}^{\infty} \alpha \varrho_{\alpha}(\theta) e^{-\lambda(\nu+s)} T\left(\frac{\nu^{\alpha}}{\theta^{\alpha}}\right) \frac{\nu^{\alpha-1}}{\theta^{\alpha}} h(s) d \nu d \theta d s \\
& =\int_{0}^{\infty} \int_{0}^{\infty} \int_{s}^{\infty} \alpha \omega_{\alpha}(\theta) e^{-\lambda \tau} T\left(\frac{(\tau-s)^{\alpha}}{\theta^{\alpha}}\right) \\
& \times \frac{(\tau-s)^{\alpha-1}}{\theta^{\alpha}} h(s) d \tau d \theta d s \quad(\tau=\nu+s)
\end{aligned}
$$

$$
\begin{aligned}
=\int_{0}^{\infty} e^{-\lambda \tau} & \\
& \times\left[\alpha \int_{0}^{\tau} \int_{0}^{\infty}{\emptyset_{\alpha}}(\theta) T\left(\frac{(\tau-s)^{\alpha}}{\theta^{\alpha}}\right)\right. \\
& \left.\times \frac{(\tau-s)^{\alpha-1}}{\theta^{\alpha}} h(s) d \theta d s\right] d \tau .
\end{aligned}
$$

According to the above work, we get

$$
\begin{array}{r}
\widehat{x}(\lambda)=\int_{0}^{\infty} e^{-\lambda t}\left[\alpha \int_{0}^{\infty} \varpi_{\alpha}(\theta) T\left(\frac{t^{\alpha}}{\theta^{\alpha}}\right) \frac{t^{\alpha-1}}{\theta^{\alpha}} x_{0} d \theta\right. \\
+\alpha \int_{0}^{t} \int_{0}^{\infty} \varpi_{\alpha}(\theta) T\left(\frac{(t-s)^{\alpha}}{\theta^{\alpha}}\right) \\
\left.\times \frac{(t-s)^{\alpha-1}}{\theta^{\alpha}} h(s) d \theta d s\right] d t .
\end{array}
$$

Now, we can invert the Laplace transform to (19) and obtain

$$
\begin{array}{r}
x(t)=\alpha \int_{0}^{\infty} \varpi_{\alpha}(\theta) T\left(\frac{t^{\alpha}}{\theta^{\alpha}}\right) \frac{t^{\alpha-1}}{\theta^{\alpha}} x_{0} d \theta \\
+\alpha \int_{0}^{t} \int_{0}^{\infty} \varpi_{\alpha}(\theta) T\left(\frac{(t-s)^{\alpha}}{\theta^{\alpha}}\right) \frac{(t-s)^{\alpha-1}}{\theta^{\alpha}} h(s) d \theta d t \\
=\alpha \int_{0}^{\infty} \frac{1}{\alpha} \theta^{-1-(1 / \alpha)} \varpi_{\alpha}\left(\theta^{-(1 / \alpha)}\right) \theta T\left(t^{\alpha} \theta\right) t^{\alpha-1} x_{0} d \theta \\
+\alpha \int_{0}^{t} \int_{0}^{\infty}(t-s)^{\alpha-1} \frac{1}{\alpha} \theta^{-1-(1 / \alpha)} \varpi_{\alpha} \\
\quad \times\left(\theta^{-(1 / \alpha)}\right) \theta T\left((t-s)^{\alpha} \theta\right) h(s) d \theta d t .
\end{array}
$$

Let

$$
\begin{gathered}
\xi_{\alpha}(\theta)=\frac{1}{\alpha} \theta^{-1-(1 / \alpha)} \varpi_{\alpha}\left(\theta^{-(1 / \alpha)}\right), \\
T_{\alpha}(t)=\alpha \int_{0}^{\infty} \theta \xi_{\alpha}(\theta) T\left(t^{\alpha} \theta\right) d \theta .
\end{gathered}
$$


Then, we get

$x(t)=t^{\alpha-1} T_{\alpha}(t) x_{0}+\int_{0}^{t}(t-s)^{\alpha-1} T_{\alpha}(t-s) h(s) d s$.

This completes the proof of the lemma.

According to Lemma 4, we give the following definition.

Definition 5. A function $x \in C_{1-\alpha}(J, X)$ is called a mild solution of (1) if it satisfies the following fractional integral equation:

$$
\begin{aligned}
x(t)= & t^{\alpha-1} T_{\alpha}(t) x_{0} \\
& +\int_{0}^{t}(t-s)^{\alpha-1} T_{\alpha}(t-s) B u(s) d s \\
& +\int_{0}^{t}(t-s)^{\alpha-1} T_{\alpha}(t-s) f(s, x(s)) d s .
\end{aligned}
$$

Remark 6. A mild solution $x(\cdot) \in C_{1-\alpha}(J, X)$ of the system (1) is referred to as a state trajectory of the fractional semilinear differential equation corresponding to the initial state $x_{0}$ and the control $u(\cdot)$.

Due to the paper [23], we can obtain the following.

Lemma 7. The operator $T_{\alpha}(t)$ has the following properties.

(i) For any fixed $t \geq 0, T_{\alpha}(t)$ is linear and bounded operators; that is, for any $x \in X$,

$$
\left\|T_{\alpha}(t) x\right\| \leq \frac{\alpha M}{\Gamma(1+\alpha)}\|x\| .
$$

(ii) $T_{\alpha}(t)(t \geq 0)$ is strongly continuous.

(iii) For any $t>0, T_{\alpha}(t)$ is also a compact operator if $T(t)$ is compact.

Let us recall the following generalized Gronwall inequality which can be found in [24].

Lemma 8. Suppose $\beta>0, a(t)$ is a nonnegative function locally integrable on $[0, \eta]$, and $b(t)$ is a nonnegative, nondecreasing continuous function defined on $[0, \eta]$ and $b(t) \leq$ $M$ (constant), and suppose $y(t)$ is nonnegative and locally integrable on $[0, \eta]$ with

$$
y(t) \leq a(t)+b(t) \int_{0}^{t}(t-s)^{\beta-1} y(s) d s, \quad t \in[0, \eta] .
$$

Then,

$$
\begin{array}{r}
y(t) \leq a(t)+\int_{0}^{t}\left[\sum_{n=1}^{\infty} \frac{[b(t) \Gamma(\beta)]^{n}}{\Gamma(n \beta)}(t-s)^{n \beta-1} a(s)\right] d s, \\
t \in[0, \eta] .
\end{array}
$$

Remark 9. Under the hypotheses of Lemma 8, let $a(t)$ be a nondecreasing function on $[0, \eta]$. Then,

$$
y(t) \leq a(t) E_{\beta}\left(b(t) \Gamma(\beta) t^{\beta}\right),
$$

where $E_{\beta}$ is the Mittag-Leffler function defined by

$$
E_{\beta}(z)=\sum_{k=0}^{\infty} \frac{z^{k}}{\Gamma(k \beta+1)} .
$$

\section{Existence of Mild Solutions}

This section is devoted to the study of the existence and uniqueness results for a class of semilinear differential equations with Riemann-Liouville fractional derivatives.

In what follows, we will make the following hypotheses on the data of our problems:

$H(1)$ : the function $f: J \times X \rightarrow X$ satisfies the following:

(i) $f(\cdot, x): J \rightarrow X$ is measurable for all $x \in X$ and $f(t, \cdot): X \rightarrow X$ is continuous for a.e. $t \in J$;

(ii) there exists a function $\phi(\cdot) \in L^{p}\left(J, R^{+}\right), p>1 / \alpha$, and a constant $\rho>0$ such that

$$
\|f(t, x(t))\| \leq \phi(t)+\rho t^{1-\alpha}\|x(t)\|, \quad \text { for a.e. } t \in J \text {; }
$$

(iii) there exists a function $\varphi(\cdot) \in L^{p}\left(J, R^{+}\right), p>1 / \alpha$, such that

$$
\|f(t, x)-f(t, y)\| \leq \varphi(t)\|x-y\|_{C_{1-\alpha}},
$$

for a.e. $t \in J$ and all $x, y \in C_{1-\alpha}(J, X)$;

$H(2)$ : the operator $B \in L_{b}\left(L^{p}(J, Y), L^{p}(J, X)\right)$;

$H(3)$ : the multivalued map $U: J \rightarrow P_{f}(Y)$ (where $P_{f}(Y)$ is a class of nonempty closed and convex subsets of $Y)$ is measurable and there exists a function $u(t) \epsilon$ $L^{p}\left(J, R^{+}\right), p>1 / \alpha$, such that

$\|U(t)\|=\sup \{\|v\|: v \in U(t)\} \leq u(t), \quad$ for a.e. $t \in J$.

Set the admissible control set

$$
\mathrm{U}_{\mathrm{ad}}=S_{U}^{p}=\left\{u \in L^{p}(J): u(t) \in U(t) \text { a.e. }\right\}, \quad p>\frac{1}{\alpha} .
$$

Then, $U_{\text {ad }} \neq \emptyset$ (see Proposition 2.1.7 and Lemma 2.3.2 of [25]). And it is not difficult to check that $U_{\text {ad }}$ is a closed and convex subset of $L^{p}(J, Y)$.

In order to discuss the solvability and optimal control of system (1), we need to consider the following.

Lemma 10. Assume that $H(1)-H(3)$ hold. Then, there exists a constant $\omega>0$ such that

$$
\|x\|_{C_{1-\alpha}} \leq \omega, \quad \text { for any solution } x \text { of }(1) .
$$

Proof. If $x$ is a mild solution of system (1) with respect to $u \in$ $U_{\text {ad }}$ on $C_{1-\alpha}(J, X)$, then

$$
\begin{aligned}
x(t)= & t^{\alpha-1} T_{\alpha}(t) x_{0}+\int_{0}^{t}(t-s)^{\alpha-1} T_{\alpha}(t-s) B u(s) d s \\
& +\int_{0}^{t}(t-s)^{\alpha-1} T_{\alpha}(t-s) f(s, x(s)) d s .
\end{aligned}
$$


For $t \in J$, we obtain that

$$
\begin{aligned}
t^{1-\alpha}\|x(t)\| \leq & \left\|T_{\alpha}(t) x_{0}\right\| \\
& +t^{1-\alpha} \int_{0}^{t}(t-s)^{\alpha-1}\left\|T_{\alpha}(t-s) B u(s)\right\| d s \\
& +t^{1-\alpha} \int_{0}^{t}(t-s)^{\alpha-1}\left\|T_{\alpha}(t-s) f(s, x(s))\right\| d s \\
\leq & \frac{\alpha M}{\Gamma(1+\alpha)} \\
& \times\left[\left\|x_{0}\right\|+t^{1-\alpha} \int_{0}^{t}(t-s)^{\alpha-1}\|(B u)(s)\| d s\right. \\
& +t^{1-\alpha} \int_{0}^{t}(t-s)^{\alpha-1} \\
& \left.\quad+\rho b^{1-\alpha} \int_{0}^{t}(t-s)^{\alpha-1} s^{1-\alpha}\|x(s)\| d s\right] \\
& \left.\frac{\alpha M}{\Gamma(1+\alpha)} \times\left[\phi(s)+\rho s^{1-\alpha}\|x(s)\|\right] d s\right] \\
& {\left[x_{0}\left\|+\left(\frac{p-1}{p \alpha-1}\right)^{(p-1) / p} b^{1-(1 / p)}+\right\| \phi L_{L^{p}}\right) } \\
& \\
& \\
& \\
& \\
& \\
& \\
& \\
& \\
& \\
&
\end{aligned}
$$

Let

$$
\begin{gathered}
\kappa=\frac{\alpha M}{\Gamma(1+\alpha)}\left[\left\|x_{0}\right\|+\left(\frac{p-1}{p \alpha-1}\right)^{(p-1) / p} b^{1-(1 / p)}\right. \\
\left.\times\left(\|B u\|_{L^{p}}+\|\phi\|_{L^{p}}\right)\right] \\
W(t)=t^{1-\alpha}\|x(t)\|
\end{gathered}
$$

then by (35), we have

$$
W(t) \leq \kappa+\frac{\alpha M \rho b^{1-\alpha}}{\Gamma(1+\alpha)} \int_{0}^{t}(t-s)^{\alpha-1} W(s) d s .
$$

It follows from Remark 9 that

$$
W(t) \leq \kappa E_{\alpha}(M \rho b):=\omega
$$

Therefore, $\|x\|_{C_{1-\alpha}}=\sup _{t \in J} t^{1-\alpha}\|x(t)\| \leq \omega$. The proof is completed.

Now, we are in the position to present our first result.
Theorem 11. Assume that the hypotheses $H(1)-H(3)$ are satisfied. Then, the problem (1) has a unique mild solution on $J$ provided that

$$
\begin{gathered}
\frac{\alpha M}{\Gamma(1+\alpha)}\left(\frac{p-1}{p \alpha-1}\right)^{1-(1 / p)} b^{1-(1 / p)}\|\varphi\|_{L^{p}}<1, \\
\frac{M b^{\alpha}}{\Gamma(1+\alpha)}<1 .
\end{gathered}
$$

Proof. Consider the operator $F: C_{1-\alpha}(J, X) \rightarrow C_{1-\alpha}(J, X)$ defined by

$$
\begin{aligned}
(F x)(t)= & t^{\alpha-1} T_{\alpha}(t) x_{0}+\int_{0}^{t}(t-s)^{\alpha-1} T_{\alpha}(t-s) B u(s) d s \\
& +\int_{0}^{t}(t-s)^{\alpha-1} T_{\alpha}(t-s) f(s, x(s)) d s .
\end{aligned}
$$

Choose

$$
\begin{aligned}
& r \geq\left(\frac { \alpha M } { \Gamma ( 1 + \alpha ) } \left[\left\|x_{0}\right\|+\left(\frac{p-1}{p \alpha-1}\right)^{(p-1) / p}\right.\right. \\
& \left.\left.\quad \times b^{\alpha-(1 / p)}\left(\|B u\|_{L^{p}}+\|\phi\|_{L^{p}}\right)\right]\right) \\
& \quad \times\left(1-\frac{M b^{\alpha}}{\Gamma(1+\alpha)}\right)^{-1},
\end{aligned}
$$

and let $B_{r}=\left\{x \in C_{1-\alpha}(J, X):\|x\| \leq r\right\}$. It is obvious that $B_{r}$ is a bounded, closed, and convex subset of $C_{1-\alpha}(J, X)$.

Firstly, we show that $F$ maps $B_{r}$ into itself. In fact, for any $x \in B_{r}$ and $t \in J$, like the proof of Lemma 10, we can easily obtain $\|(F x)(t)\| \leq r$ which means that $F B_{r} \subseteq B_{r}$.

Next, we show that $F$ is a contraction operator on $C_{1-\alpha}(J, X)$.

Indeed, let $t \in J$, and $x, y \in C_{1-\alpha}(J, X)$; then we obtain

$$
\begin{aligned}
& t^{1-\alpha}\|(F x)(t)-(F y)(t)\| \\
& \leq t^{1-\alpha} \int_{0}^{t}(t-s)^{\alpha-1} \\
& \quad \times\left\|T_{\alpha}(t-s)[f(s, x(s))-f(s, y(s))]\right\| d s \\
& \leq \frac{\alpha M}{\Gamma(1+\alpha)} t^{1-\alpha} \int_{0}^{t}(t-s)^{\alpha-1} \varphi(s) \\
& \quad \times\|x-y\|_{C_{1-\alpha}} d s \\
& \leq \frac{\alpha M}{\Gamma(1+\alpha)}\left(\frac{p-1}{p \alpha-1}\right)^{1-(1 / p)} b^{1-(1 / p)} \\
& \quad \times\|\varphi\|_{L^{p}}\|x-y\|_{C_{1-\alpha}} .
\end{aligned}
$$

Since $(\alpha M /(\Gamma(1+\alpha)))((p-1) /(p \alpha-$ $1))^{1-(1 / p)} b^{1-(1 / p)}\|\varphi\|_{L^{p}}<1$, so $F$ is a contradiction operator. According to Banach's fixed point theorem, we obtain the problem (1) that has a unique mild solution on $J$. The proof is completed. 
Remark 12. In Theorem 11, we investigate the existence of a local mild solution of (1). Next, if we assume that the semigroup $T(t)(t>0)$ is compact, then we can get the global version of a mild solution for system (1).

Firstly, for a compact semigroup, we have the following.

Lemma 13 (see [26]). Let $T(t)$ be a $C_{0}$-semigroup. If $T(t)$ is a compact semigroup, then $T(t)$ is continuous in the uniform operator topology for $t>0$.

The key tool in the existence result is the following Schaefer fixed point theorem.

Theorem 14. Let $X$ be a Banach space and let $F: X \rightarrow X$ be a completely continuous operator. If the set

$$
\Omega(F)=\{x \in X: x=\lambda F(x), \lambda \in(0,1)\}
$$

is bounded, then $F$ has at least a fixed point.

Now, we are ready to state the existence result which is based on Theorem 14.

Theorem 15. Assume that $H(1)-H(3)$ hold; if $A$ is the infinitesimal generator of a compact semigroup $T(t)$, then the problem (1) has at least one mild solution on J.

Proof. We consider the operator $F: C_{1-\alpha}(J, X) \rightarrow$ $C_{1-\alpha}(J, X)$ defined by

$$
\begin{aligned}
(F x)(t)= & t^{\alpha-1} T_{\alpha}(t) x_{0}+\int_{0}^{t}(t-s)^{\alpha-1} T_{\alpha}(t-s) B u(s) d s \\
& +\int_{0}^{t}(t-s)^{\alpha-1} T_{\alpha}(t-s) f(s, x(s)) d s .
\end{aligned}
$$

For the sake of convenience, we subdivide the proof into several steps.

Step 1. F maps bounded sets into bounded sets in $C_{1-\alpha}(J, X)$.

In fact, it is enough to show that, for any $r>0$, there exists a $\eta>0$ such that, for each $x \in B_{r}=\left\{x \in C_{1-\alpha}(J, X)\right.$ : $\left.\|x\|_{C_{1-\alpha}} \leq r\right\}$, we have $\|F x\|_{C_{1-\alpha}} \leq \eta$.

For each $t \in J$, we obtain

$$
\begin{aligned}
& t^{1-\alpha}\left\|\left(F^{x}\right)(t)\right\| \leq\left\|T_{\alpha}(t) x_{0}\right\| \\
&+t^{1-\alpha} \int_{0}^{t}(t-s)^{\alpha-1}\left\|T_{\alpha}(t-s)(B u)(s)\right\| d s \\
&+t^{1-\alpha} \int_{0}^{t}(t-s)^{\alpha-1}\left\|T_{\alpha}(t-s) f(s, x(s))\right\| d s \\
& \leq \frac{\alpha M}{\Gamma(1+\alpha)} \\
& \times\left[\left\|x_{0}\right\|+t^{1-\alpha} \int_{0}^{t}(t-s)^{\alpha-1}\|(B u)(s)\| d s\right. \\
&+t^{1-\alpha} \int_{0}^{t}(t-s)^{\alpha-1} \\
&\left.\times\left[\phi(s)+\rho s^{1-\alpha}\|x(s)\|\right] d s\right]
\end{aligned}
$$

$$
\begin{aligned}
& \leq \frac{\alpha M}{\Gamma(1+\alpha)} \\
& \quad \times\left[\left\|x_{0}\right\|+\left(\frac{p-1}{p \alpha-1}\right)^{(p-1) / p} b^{1-(1 / p)}\right. \\
& \left.\quad \quad \times\left(\|B u\|_{L^{p}}+\|\phi\|_{L^{p}}\right)\right]+\frac{\rho M b r}{\Gamma(1+\alpha)},
\end{aligned}
$$

which implies that

$$
\begin{aligned}
\|F x\|_{C_{1-\alpha}} \leq & \frac{\alpha M}{\Gamma(1+\alpha)} \\
& \times\left[\left\|x_{0}\right\|+\left(\frac{p-1}{p \alpha-1}\right)^{(p-1) / p}\right. \\
& \left.\quad \times b^{1-(1 / p)}\left(\|B u\|_{L^{p}}+\|\phi\|_{L^{p}}\right)\right] \\
& +\frac{\rho M b r}{\Gamma(1+\alpha)}:=\eta .
\end{aligned}
$$

Thus, we know that $\left\{t^{1-\alpha}\left(F^{x}\right)(t): x \in B_{r}\right\}$ is a bounded set in $C(J, X)$.

Step 2. We prove that $F$ is continuous.

Let $\left\{x_{n}\right\}$ be a sequence such that $x_{n} \rightarrow x$ in $C_{1-\alpha}(J, X)$ as $n \rightarrow \infty$. Then, for each $t \in J$, we obtain

$$
\begin{aligned}
& t^{1-\alpha}\left\|\left(F x_{n}\right)(t)-(F x)(t)\right\| \\
& \leq t^{1-\alpha} \int_{0}^{t}(t-s)^{\alpha-1} \| T_{\alpha}(t-s) \\
& \quad \times\left[f\left(s, x_{n}(s)\right)-f(s, x(s))\right] \| d s \\
& \leq \frac{\alpha M}{\Gamma(1+\alpha)} t^{1-\alpha} \\
& \quad \times \int_{0}^{t}(t-s)^{\alpha-1} \varphi(s)\left\|x_{n}-x\right\|_{C_{1-\alpha}} d s \\
& \leq \frac{\alpha M}{\Gamma(1+\alpha)} \\
& \quad \times\left(\frac{p-1}{p \alpha-1}\right)^{1-(1 / p)} b^{1-(1 / p)}\|\varphi\|_{L^{p}}\left\|x_{n}-x\right\|_{C_{1-\alpha}} .
\end{aligned}
$$

Hence, we get

$$
\begin{aligned}
\left\|F x_{n}-F x\right\|_{C_{1-\alpha}} \leq & \frac{\alpha M}{\Gamma(1+\alpha)} \\
& \times\left(\frac{p-1}{p \alpha-1}\right)^{1-(1 / p)} b^{1-(1 / p)} \\
& \times\|\varphi\|_{L^{p}}\left\|x_{n}-x\right\|_{C_{1-\alpha}} \longrightarrow 0 \quad \text { as } n \longrightarrow \infty .
\end{aligned}
$$

Step 3. We prove that $\left\{t^{1-\alpha}(F x)(t): x \in B_{r}\right\}$ is an equicontinuous set in $C(J, X)$. 
Firstly, for any $\varepsilon>0$, by Lemma 7(ii), $T_{\alpha}(t)$ is strongly continuous; then, there exists a $\delta^{\prime}>0$, such that

$$
\left\|T_{\alpha}\left(\tau_{2}\right) x_{0}-T_{\alpha}\left(\tau_{1}\right) x_{0}\right\|<\frac{\varepsilon}{2} \text {, as }\left|\tau_{2}-\tau_{1}\right|<\delta^{\prime} .
$$

Thus, for the above $\varepsilon>0$, there exists $\delta_{0}=\min \left\{\delta^{\prime},((p \alpha-\right.$ 1)/(p-1)) $\left.\left[\Gamma(\alpha) \varepsilon /\left(2 M\left(\|B u\|_{L^{p}}+\|\phi\|_{L^{p}}+\rho r\right)\right)\right]^{p /(p-1)}\right\}>0$, such that, for any $x \in B_{r}, \tau_{1}=0,0<\tau_{2} \leq \delta_{0}$, one can obtain

$$
\begin{aligned}
& \left\|\tau_{2}^{1-\alpha}(F x)\left(\tau_{2}\right)-\tau_{1}^{1-\alpha}(F x)\left(\tau_{1}\right)\right\| \\
& \leq\left\|T_{\alpha}\left(\tau_{2}\right) x_{0}-x_{0}\right\| \\
& \quad+\left\|\tau_{2}^{1-\alpha} \int_{0}^{\tau_{2}}\left(\tau_{2}-s\right)^{\alpha-1} T_{\alpha}\left(\tau_{2}-s\right) B u(s) d s\right\| \\
& \quad+\left\|\tau_{2}^{1-\alpha} \int_{0}^{\tau_{2}}\left(\tau_{2}-s\right)^{\alpha-1} T_{\alpha}\left(\tau_{2}-s\right) f(s, x(s)) d s\right\| \\
& \leq\left\|T_{\alpha}\left(\tau_{2}\right) x_{0}-x_{0}\right\|+\frac{\alpha M}{\Gamma(1+\alpha)}\left(\frac{p-1}{p \alpha-1}\right)^{(p-1) / p} \\
& \quad \times\left(\|B u\|_{L^{p}}+\|\phi\|_{L^{p}}+\rho r\right) \delta_{0}^{1-(1 / p)} \\
& <\frac{\varepsilon}{2}+\frac{\varepsilon}{2}=\varepsilon .
\end{aligned}
$$

Hence, by the definition of equicontinuity, we get that $F$ is equicontinuous on $\left[0, \delta_{0}\right]$.

Next, for any $x \in B_{r}$ and $\left(\delta_{0} / 2\right) \leq \tau_{1}<\tau_{2} \leq b$, we obtain

$$
\begin{aligned}
& \left\|\tau_{2}^{1-\alpha}(F x)\left(\tau_{2}\right)-\tau_{1}^{1-\alpha}(F x)\left(\tau_{1}\right)\right\| \\
& \leq\left\|T_{\alpha}\left(\tau_{2}\right) x_{0}-T_{\alpha}\left(\tau_{1}\right) x_{0}\right\| \\
& +\| \tau_{2}^{1-\alpha} \int_{0}^{\tau_{2}}\left(\tau_{2}-s\right)^{\alpha-1} T_{\alpha}\left(\tau_{2}-s\right) B u(s) d s \\
& \quad-t^{1-\alpha} \int_{0}^{\tau_{1}}\left(\tau_{1}-s\right)^{\alpha-1} T_{\alpha}\left(\tau_{1}-s\right) B u(s) d s \| \\
& +\| \tau_{2}^{1-\alpha} \int_{0}^{\tau_{2}}\left(\tau_{2}-s\right)^{\alpha-1} \\
& \quad \times T_{\alpha}\left(\tau_{2}-s\right) f(s, x(s)) d s-\tau_{1}^{1-\alpha} \\
& \leq\left\|T_{\alpha}^{\tau_{1}}\left(\tau_{2}\right) x_{0}-T_{\alpha}\left(\tau_{1}\right) x_{0}\right\| \\
& +\left[\tau_{2}^{1-\alpha}-\tau_{1}^{1-\alpha}\right]\left\|\int_{0}^{\tau_{1}}\left(\tau_{1}-s\right)^{\alpha-1} T_{\alpha}\left(\tau_{1}-s\right) B u(s) d s\right\| \\
& +\tau_{2}^{1-\alpha} \| \int_{0}^{\tau_{1}}\left[\left(\tau_{1}-s\right)^{\alpha-1}-\left(\tau_{2}-s\right)^{\alpha-1}\right] \\
& \quad \times T_{\alpha}\left(\tau_{1}-s\right) B u(s) d s \| \\
& +\tau_{2}^{1-\alpha} \| \int_{0}^{\tau_{1}}\left(\tau_{1}-s\right)^{\alpha-1}\left[T_{\alpha}\left(\tau_{2}-s\right)\right. \\
& \left.\quad-T_{\alpha}\left(\tau_{1}-s\right)\right] B u(s) d s \|
\end{aligned}
$$

$$
\begin{aligned}
& +\tau_{2}^{1-\alpha}\left\|\int_{\tau_{1}}^{\tau_{2}}\left(\tau_{2}-s\right)^{\alpha-1} T_{\alpha}\left(\tau_{2}-s\right) B u(s) d s\right\| \\
& +\left[\tau_{2}^{1-\alpha}-\tau_{1}^{1-\alpha}\right] \| \int_{0}^{\tau_{1}}\left(\tau_{1}-s\right)^{\alpha-1} \\
& \quad \times T_{\alpha}\left(\tau_{1}-s\right) f(s, x(s)) d s \| \\
& +\tau_{2}^{1-\alpha} \| \int_{0}^{\tau_{1}}\left[\left(\tau_{1}-s\right)^{\alpha-1}-\left(\tau_{2}-s\right)^{\alpha-1}\right] \\
& \quad \times T_{\alpha}\left(\tau_{1}-s\right) f(s, x(s)) d s \| \\
& +\tau_{2}^{1-\alpha} \| \int_{0}^{\tau_{1}}\left(\tau_{1}-s\right)^{\alpha-1}\left[T_{\alpha}\left(\tau_{2}-s\right)\right. \\
& \left.\quad-T_{\alpha}\left(\tau_{1}-s\right)\right] f(s, x(s)) d s \| \\
& \leq \tau_{2}^{1-\alpha}\left\|\int_{\tau_{1}}^{\tau_{2}}\left(\tau_{2}-s\right)^{\alpha-1} T_{\alpha}\left(\tau_{2}-s\right) f(s, x(s)) d s\right\|
\end{aligned}
$$

By the assumptions and Holder's inequality, we have

$$
\begin{aligned}
Q_{2} \leq & {\left[\tau_{2}^{1-\alpha}-\tau_{1}^{1-\alpha}\right] \frac{\alpha M}{\Gamma(1+\alpha)} } \\
& \times \int_{0}^{\tau_{1}}\left(\tau_{1}-s\right)^{\alpha-1}\|B u(s)\| d s \\
\leq & \frac{\alpha M}{\Gamma(1+\alpha)}\left(\frac{p-1}{p \alpha-1}\right)^{(p-1) / p} \\
& \quad \times\|B u\|_{L^{p}} \tau_{1}^{\alpha-(1 / p)}\left[\tau_{2}^{1-\alpha}-\tau_{1}^{1-\alpha}\right] .
\end{aligned}
$$

Similarly, we obtain

$$
\begin{aligned}
& Q_{3} \leq \frac{2 \alpha M \tau_{2}^{1-\alpha}}{\Gamma(1+\alpha)}\left(\frac{p-1}{p \alpha-1}\right)^{(p-1) / p} \\
& \times\|B u\|_{L^{p}}\left(\tau_{2}-\tau_{1}\right)^{\alpha-(1 / p)}, \\
& Q_{5} \leq \frac{\alpha M \tau_{2}^{1-\alpha}}{\Gamma(1+\alpha)}\left(\frac{p-1}{p \alpha-1}\right)^{(p-1) / p} \\
& \times\|B u\|_{L^{p}}\left(\tau_{2}-\tau_{1}\right)^{\alpha-(1 / p)}, \\
& Q_{6} \leq \frac{\alpha M}{\Gamma(1+\alpha)}\left(\frac{p-1}{p \alpha-1}\right)^{1-(1 / p)} \\
& \times {\left[\|\phi\|_{L^{p}}+\rho \omega\right] \tau_{1}^{\alpha-(1 / p)}\left[\tau_{2}^{1-\alpha}-\tau_{1}^{1-\alpha}\right], } \\
& Q_{7} \leq \frac{2 \alpha M \tau_{2}^{1-\alpha}}{\Gamma(1+\alpha)}\left(\frac{p-1}{p \alpha-1}\right)^{1-(1 / p)} \\
& \quad \times\left[\|\phi\|_{L^{p}}+\rho \omega\right]\left(\tau_{2}-\tau_{1}\right)^{\alpha-(1 / p)}, \\
& Q_{9} \leq \frac{\alpha M \tau_{2}^{1-\alpha}}{\Gamma(1+\alpha)}\left(\frac{p-1}{p \alpha-1}\right)^{1-(1 / p)} \\
& \times\left[\|\phi\|_{L^{p}}+\rho \omega\right]\left(\tau_{2}-\tau_{1}\right)^{\alpha-(1 / p)},
\end{aligned}
$$


and, for $Q_{4}, Q_{8}$, there exists a $\delta>0$ which is small enough, such that

$$
\begin{aligned}
& Q_{4} \leq \tau_{2}^{1-\alpha}\left[\| \int_{0}^{\tau_{1}-\delta}\left(\tau_{1}-s\right)^{\alpha-1}\left[T_{\alpha}\left(\tau_{2}-s\right)\right.\right. \\
& \left.-T_{\alpha}\left(\tau_{1}-s\right)\right] B u(s) d s \| \\
& +\| \int_{\tau_{1}-\delta}^{\tau_{1}}\left(\tau_{1}-s\right)^{\alpha-1}\left[T_{\alpha}\left(\tau_{2}-s\right)\right. \\
& \left.\left.-T_{\alpha}\left(\tau_{1}-s\right)\right] B u(s) d s \|\right] \\
& \leq \sup _{s \in\left[0, \tau_{1}-\delta\right]} \| T_{\alpha}\left(\tau_{2}-s\right) \\
& -T_{\alpha}\left(\tau_{1}-s\right) \| \tau_{2}^{1-\alpha}\left(\frac{p-1}{p \alpha-1}\right)^{(p-1) / p} \\
& \times\|B u\|_{L^{p}}\left(\tau_{1}^{(\alpha p-1) /(p-1)}-\delta^{(\alpha p-1) /(p-1)}\right)^{1-(1 / p)} \\
& +\frac{2 \alpha M \tau_{2}^{1-\alpha}}{\Gamma(1+\alpha)}\left(\frac{p-1}{p \alpha-1}\right)^{(p-1) / p}\|B u\|_{L^{p}} b^{1-\alpha} \delta^{1-(1 / p)}, \\
& Q_{8} \leq \sup _{s \in\left[0, \tau_{1}-\delta\right]}\left\|T_{\alpha}\left(\tau_{2}-s\right)-T_{\alpha}\left(\tau_{1}-s\right)\right\| \\
& \times \tau_{2}^{1-\alpha}\left(\frac{p-1}{p \alpha-1}\right)^{(p-1) / p}\left[\|\phi\|_{L^{p}}+\rho \omega\right] \\
& \times\left(\tau_{1}^{(\alpha p-1) /(p-1)}-\delta^{(\alpha p-1) /(p-1)}\right)^{1-(1 / p)} \\
& +\frac{2 \alpha M \tau_{2}^{1-\alpha}}{\Gamma(1+\alpha)}\left(\frac{p-1}{p \alpha-1}\right)^{(p-1) / p}\left[\|\phi\|_{L^{p}}+\rho \omega\right] b^{1-\alpha} \delta^{1-(1 / p)} .
\end{aligned}
$$

Since the compactness of $T(t)(t>0)$ and Lemma 13 imply the continuity of $T_{\alpha}(t)(t>0)$ in $t$ in the uniform operator topology, it can be easily seen that $Q_{4}$ and $Q_{8}$ tend to zero independently of $x \in B_{r}$ as $\tau_{2} \rightarrow \tau_{1}, \delta \rightarrow 0$. It is also clear that $Q_{i}(i=1,2,3,5,6,7,9)$ tend to zero as $\tau_{2} \rightarrow \tau_{1}$ does not depend on particular choice of $x$. Thus, we get that $\left\|\tau_{2}^{1-\alpha}(F x)\left(\tau_{2}\right)-\tau_{1}^{1-\alpha}(F x)\left(\tau_{1}\right)\right\|$ tends to zero independently of $x \in B_{r}$ as $\tau_{2} \rightarrow \tau_{1}$ which implies that $F$ is equicontinuous on $\delta_{0} / 2 \leq \tau_{1}<\tau_{2} \leq b$.

Therefore, by all of the above work, we can get that $\left\{t^{1-\alpha}(F x)(t): x \in B_{r}\right\}$ is an equicontinuous set in $C(J, X)$.

Step 4. We show that $F$ is compact.

Let $t \in J$ be fixed; we show that the set $\Pi(t)=$ $\left\{t^{1-\alpha}(F x)(t): x \in B_{r}\right\}$ is relatively compact in $X$.

Clearly, $\Pi(0)=\left\{x_{0}\right\}$ is compact, so it is only necessary to consider $t>0$. For each $\epsilon \in(0, t), t \in(0, b]$, and $x \in B_{r}$ and any $\delta>0$, we define

$$
\Pi_{\epsilon, \delta}(t)=\left\{t^{1-\alpha} F_{\epsilon, \delta}(x)(t): x \in B_{r}\right\},
$$

where

$$
\begin{aligned}
& t^{1-\alpha} F_{\epsilon, \delta}(x)(t) \\
& \quad=T_{\alpha}(t) x_{0}+\alpha t^{1-\alpha}
\end{aligned}
$$

$$
\begin{gathered}
\times \int_{0}^{t-\epsilon} \int_{\delta}^{\infty} \theta(t-s)^{\alpha-1} \xi_{\alpha}(\theta) T\left((t-s)^{\alpha} \theta\right) \\
\times[B u(s)+f(s, x(s))] d \theta d s . \\
=T_{\alpha}(t) x_{0}+\alpha T\left(\epsilon^{\alpha} \delta\right) t^{1-\alpha} \\
\times \int_{0}^{t-\epsilon} \int_{\delta}^{\infty} \theta(t-s)^{\alpha-1} \xi_{\alpha}(\theta) T\left((t-s)^{\alpha} \theta-\epsilon^{\alpha} \delta\right) \\
\times[B u(s)+f(s, x(s))] d \theta d s .
\end{gathered}
$$

From the boundedness of $\int_{0}^{t-\epsilon} \int_{\delta}^{\infty} \theta(t-s)^{\alpha-1} \xi_{\alpha}(\theta) T((t-$ $\left.s)^{\alpha} \theta-\epsilon^{\alpha} \delta\right)[B u(s)+f(s, x(s))] d \theta d s$ and the compactness of $T\left(\epsilon^{\alpha} \delta\right)\left(\epsilon^{\alpha} \delta>0\right)$, we obtain that the set $\Pi_{\epsilon, \delta}(t)=$ $\left\{t^{1-\alpha} F_{\epsilon, \delta}(x)(t): x \in B_{r}\right\}$ is relatively compact set in $X$ for each $\epsilon \in(0, t)$ and $\delta>0$. Moreover, we have

$$
\begin{gathered}
t^{1-\alpha}\left\|F(x)(t)-F_{\epsilon, \delta}(x)(t)\right\| \\
=t^{1-\alpha} \| \alpha \int_{0}^{t} \int_{0}^{\infty} \theta(t-s)^{\alpha-1} \xi_{\alpha}(\theta) T\left((t-s)^{\alpha} \theta\right) \\
\times[B u(s)+f(s, x(s))] d \theta d s \\
-\alpha \int_{0}^{t-\epsilon} \int_{\delta}^{\infty} \theta(t-s)^{\alpha-1} \xi_{\alpha}(\theta) T\left((t-s)^{\alpha} \theta\right) \\
\times[B u(s)+f(s, x(s))] d \theta d s \| \\
\leq \alpha t^{1-\alpha} \| \int_{0}^{t} \int_{0}^{\delta} \theta(t-s)^{\alpha-1} \xi_{\alpha}(\theta) T\left((t-s)^{\alpha} \theta\right) \\
\times[B u(s)+f(s, x(s))] d \theta d s \| \\
+\alpha t^{1-\alpha} \| \int_{t-\epsilon}^{t} \int_{\delta}^{\infty} \theta(t-s)^{\alpha-1} \xi_{\alpha}(\theta) T\left((t-s)^{\alpha} \theta\right) \\
\times\left[b^{1-(1 / p)} \int_{0}^{\delta} \theta \xi_{\alpha}(\theta) d \theta+\frac{b^{1-\alpha}}{\Gamma(1+\alpha)} \epsilon^{\alpha-(1 / p)}\right] . \\
\leq \alpha M\left(\frac{p-1}{p \alpha-1}\right)^{1-(1 / p)}\left[\|B u\|_{\left.L^{p}+\| \phi L_{L^{p}}+r \rho\right]} \times[(s u(s, x(s))] d \theta d s \|\right.
\end{gathered}
$$

Since $\int_{0}^{\infty} \theta \xi_{\alpha}(\theta) d \theta=1 /(\Gamma(1+\alpha))$, the last inequality tends to zero when $\epsilon \rightarrow 0$ and $\delta \rightarrow 0$. Therefore, there are relatively compact sets arbitrarily close to the set $\Pi(t)(t>0)$. Hence, the set $\Pi(t)(t>0)$ is also relatively compact in $X$.

Step 5. A priori bounds.

Now, it remains to show that the set

$$
\Omega(F)=\left\{x \in C_{1-\alpha}(J, X): x=\lambda_{F}(x), \lambda \in(0,1)\right\}
$$

is bounded.

Let $x \in \Omega(F)$; then $x=\lambda_{F}(x)$ for some $\lambda \in(0,1)$. Similarly to the proof of Lemma 10 , we obtain

$$
\|x\|_{C_{1-\alpha}} \leq \kappa E_{\alpha}(M \rho b) \text {. }
$$

This shows that the set $\Omega(F)$ is bounded. 
As a result, by the conclusion of Theorem 14, we obtain that $F$ has a fixed point $x$. Therefore, system (1) has at least one mild solution on $J$. The proof is completed.

\section{Optimal Control Results}

In this section, we are concerned with the following Lagrange problem (P).

Minimize a cost function of the form

$$
\mathscr{J}(x, u):=\int_{0}^{b} \mathscr{L}(t, x(t), u(t)) d t
$$

among all the admissible state control pairs of the semilinear differential equations with Riemann-Liouville fractional derivatives (1); that is, find an admissible state control pair $\left(x^{0}, u^{0}\right) \in C_{1-\alpha}(J, X) \times U_{\text {ad }}$ such that

$$
\mathscr{J}\left(x^{0}, u^{0}\right) \leq \mathscr{J}(x, u), \quad \forall(x, u) \in C_{1-\alpha}(J, X) \times U_{\text {ad }},
$$

where $x$ denotes the mild solution of system (1) corresponding to the control $u \in U_{\text {ad }}$.

For the existence of solution for problem (P), we will introduce the following assumptions:

$H(4)$ : the function $\mathscr{L}: J \times X \times Y \rightarrow R \cup\{\infty\}$ satisfies the following:

(i) the function $\mathscr{L}: J \times X \times Y \rightarrow R \cup\{\infty\}$ is Borel measurable;

(ii) $\mathscr{L}(t, \cdot, \cdot)$ is sequentially lower semicontinuous on $X \times$ $Y$ for almost all $t \in J$;

(iii) $\mathscr{L}(t, x, \cdot)$ is convex on $Y$ for each $x \in X$ and almost all $t \in J$

(iv) there exist constants $c \geq 0, d>0, \psi$ is nonnegative, and $\psi \in L^{1}(J, R)$ such that

$$
\mathscr{L}(t, x, u) \geq \psi(t)+c\|x\|_{X}+d\|u\|_{Y} .
$$

Next, we can give the following result on existence of optimal controls for problem (P).

Theorem 16. Let the assumptions of Theorem 15 and $H(4)$ hold. Then, Lagrange problem $(P)$ admits an optimal pair; that is, there exists an admissible control pair $\left(x^{0}, u^{0}\right) \in$ $C_{1-\alpha}(J, X) \times U_{a d}$ such that

$$
\begin{array}{r}
\mathscr{J}\left(x^{0}, u^{0}\right)=\int_{0}^{b} \mathscr{L}\left(t, x^{0}(t), u^{0}(t)\right) d t \leq \mathscr{J}(x, u), \\
\forall(x, u) \in C_{1-\alpha}(J, X) \times U_{a d} .
\end{array}
$$

Proof. If $\inf \left\{\mathscr{J}(x, u):(x, u) \in C_{1-\alpha}(J, X) \times U_{\text {ad }}\right\}=+\infty$, then it is clear that the Lagrange problem $(\mathrm{P})$ has an optimal pair.

Without loss of generality, we assume that $\inf \{\mathscr{J}(x, u)$ : $\left.(x, u) \in C_{1-\alpha}(J, X) \times U_{\text {ad }}\right\}=\xi<+\infty$. Using $H(4)$ (iv), we have $\xi>-\infty$. By definition of infimum, there exists a minimizing sequence feasible pair $\left\{\left(x^{m}, u^{m}\right)\right\} \subset \mathscr{P}_{\text {ad }} \equiv\{(x, u): x$ that is a mild solution of system (1) corresponding to $\left.u \in U_{\text {ad }}\right\}$, such that $\mathscr{J}\left(x^{m}, u^{m}\right) \rightarrow \xi$ as $m \rightarrow+\infty$. Since $\left\{u^{m}\right\} \subseteq U_{\text {ad }}(m=$
$1,2, \ldots),\left\{u^{m}\right\}$ is a bounded subset of the separable reflexive Banach space $L^{p}(J, Y)$, there exists a subsequence, relabeled as $\left\{u^{m}\right\}$, and $u^{0} \in L^{p}(J, Y)$ such that

$$
u^{m} \rightarrow u^{0} \quad \text { in } L^{p}(J, Y) .
$$

Since $U_{\mathrm{ad}}$ is closed and convex, by Mazur's lemma, $u^{0} \epsilon$ $U_{\text {ad }}$.

Let $\left\{x^{m}\right\}$ denote the sequence of solutions of the system (1) corresponding to $\left\{u^{m}\right\}$; that is,

$$
\begin{aligned}
x^{m}(t)= & t^{\alpha-1} T_{\alpha}(t) x_{0}+\int_{0}^{t}(t-s)^{\alpha-1} T_{\alpha}(t-s) B u^{m}(s) d s \\
& +\int_{0}^{t}(t-s)^{\alpha-1} T_{\alpha}(t-s) f\left(s, x^{m}(s)\right) d s .
\end{aligned}
$$

Now, we prove that $\left\{x^{m}\right\}$ is relatively compact on $C_{1-\alpha}(J, X)$.

Firstly, it follows the boundedness of $\left\{u^{m}\right\}$ and Lemma 10, and one can check that there exists a positive number $\omega$ such that $\left\|x^{m}\right\|_{C_{1-\alpha}} \leq \omega$, which implies that $\left\|x^{m}\right\|_{C_{1-\alpha}}$ is uniformly bounded.

Next, denote $g^{m}(t)=t^{1-\alpha} x^{m}(t)$; like the proof of Steps 3 and 4 in Theorem 15, $\left\{g: g=t^{1-\alpha} x(t)\right\}$ is equicontinuous and relatively compact subsets of $C(J, X)$. Hence, we can deduce that $\left\{x^{m}\right\}$ is relatively compact on $C_{1-\alpha}(J, X)$. Therefore, there exists a function $x^{0} \in C_{1-\alpha}(J, X)$ such that

$$
x^{m} \longrightarrow x^{0} \text { in } C_{1-\alpha}(J, X) \text {. }
$$

Moreover, by $H(1)($ iii), we get

$$
\left\|f\left(t, x^{m}(t)\right)-f\left(t, x^{0}(t)\right)\right\| \leq \varphi(t)\left\|x^{m}-x^{0}\right\|_{C_{1-\alpha}},
$$

and then, in view of (66), we can obtain

$$
f\left(t, x^{m}(t)\right) \longrightarrow f\left(t, x^{0}(t)\right), \quad \text { a.e. } t \in J .
$$

And by $H(1)(\mathrm{ii})$, we get

$$
\left\|f\left(t, x^{m}(t)\right)\right\| \leq \phi(t)+\rho t^{1-\alpha}\|x(t)\| \leq \phi(t)+\rho \omega .
$$

Thus, by applying the dominated convergence theorem, one can prove that

$$
\begin{aligned}
& \int_{0}^{t}(t-s)^{\alpha-1} T_{\alpha}(t-s) f\left(s, x^{m}(s)\right) d s \\
& \quad \longrightarrow \int_{0}^{t}(t-s)^{\alpha-1} T_{\alpha}(t-s) f\left(s, x^{0}(s)\right) d s, \quad \text { a.e. } t \in J .
\end{aligned}
$$

Similarly, we have

$$
\begin{aligned}
& \int_{0}^{t}(t-s)^{\alpha-1} T_{\alpha}(t-s) B u^{m}(s) d s \\
& \quad \longrightarrow \int_{0}^{t}(t-s)^{\alpha-1} T_{\alpha}(t-s) B u^{0}(s) d s, \quad \text { a.e. } t \in J .
\end{aligned}
$$


Hence, it follows from (65) that

$$
\begin{aligned}
x^{0}(t)= & t^{\alpha-1} T_{\alpha}(t) x_{0}+\int_{0}^{t}(t-s)^{\alpha-1} T_{\alpha}(t-s) B u^{0}(s) d s \\
& +\int_{0}^{t}(t-s)^{\alpha-1} T_{\alpha}(t-s) f\left(s, x^{0}(s)\right) d s ;
\end{aligned}
$$

that is, $x^{0}$ denotes the sequence of solutions of the system (1) corresponding to $u^{0}$.

Note that $H(4)$ implies that all of the assumptions of Balder (see Theorem 2.1 [27]) are satisfied. Hence, by Balder's theorem, we can conclude that $(x, u) \rightarrow \int_{0}^{b} \mathscr{L}(t, x(t), u(t)) d t$ is sequentially lower semicontinuous in the strong topology of $L^{1}(J, X) \times L^{1}(J, Y)$. Since $L^{p}(J, X) \times L^{p}(J, Y) \subset L^{1}(J, X) \times$ $L^{1}(J, Y), \mathcal{J}$ is also sequentially lower semicontinuous in $L^{p}(J, X) \times L^{p}(J, Y)$. Hence, $\mathscr{J}$ is weakly lower semicontinuous on $L^{p}(J, X) \times L^{p}(J, Y)$, and since, by $H(4)($ iv $), \mathscr{J}>-\infty, \mathcal{J}$ attains its infimum at $\left(x^{0}, u^{0}\right) \in C_{1-\alpha}(J, X) \times U_{\text {ad }}$; that is,

$$
\begin{aligned}
\xi & =\lim _{m \rightarrow \infty} \int_{0}^{b} \mathscr{L}\left(t, x^{m}(t), u^{m}(t)\right) d t \\
& \geq \int_{0}^{b} \mathscr{L}\left(t, x^{0}(t), u^{0}(t)\right) d t=J\left(x^{0}, u^{0}\right) \geq \xi .
\end{aligned}
$$

The proof is completed.

\section{An Example}

Consider the following initial-boundary value problem of fractional parabolic control system with Riemann-Liouville fractional derivatives:

$$
\begin{aligned}
{ }^{L} D_{t}^{\alpha} x(t, y)= & \frac{\partial^{2}}{\partial y^{2}} x(t, y)+e^{-t}+\frac{t^{1-\alpha}}{(t+6)^{2}} \sin (x(t, y)) \\
& +\int_{0}^{1} q(y, \tau) u(\tau, t) d \tau, \\
& t \in J=[0,1], \quad y \in[0, \pi], \quad u \in U_{\mathrm{ad}}, \\
x(t, 0) & =x(t, \pi)=0, \quad t \in J=[0,1], \\
\left.I_{0^{+}}^{1-\alpha} x(t, y)\right|_{t=0} & =x_{0}(y), \quad t \in[0,1], y \in[0, \pi],
\end{aligned}
$$

with the cost function

$$
\mathscr{J}(x, u)=\int_{0}^{1} \int_{0}^{\pi}|x(t, y)|^{2} d y d t+\int_{0}^{1} \int_{0}^{\pi}|u(t, y)|^{2} d y d t,
$$

where $\alpha=2 / 3, q:[0,1] \times[0,1] \rightarrow R$ is continuous, and $u \in L^{2}(J,[0, \pi])$.

Take $X=Y=L^{2}(J,[0, \pi])$ and the operator $A: D(A) \subset$ $X \rightarrow X$ is defined by

$$
A \omega=\omega^{\prime \prime}
$$

where the domain $D(A)$ is given by

$$
\left\{\omega \in X: \omega, \omega^{\prime}\right. \text { are absolutely continuous, }
$$$$
\left.\omega^{\prime \prime} \in X, \omega(0)=\omega(\pi)=0\right\} .
$$

Then, $A$ can be written as

$$
A \omega=\sum_{n=1}^{\infty} n^{2}\left(\omega, \omega_{n}\right) \omega_{n}, \quad \omega \in D(A),
$$

where $\omega_{n}(x)=\sqrt{2 / \pi} \sin n x(n=1,2, \ldots)$ is an orthonormal basis of $X$. It is well known that $A$ is the infinitesimal generator of a compact semigroup $T(t)(t>0)$ in $X$ given by

$$
\begin{gathered}
T(t) x=\sum_{n=1}^{\infty} \exp ^{-n^{2} t}\left(x, x_{n}\right) x_{n}, \quad x \in X, \\
\|T(t)\| \leq e^{-1}<1=M .
\end{gathered}
$$

We take the functions $u: \Phi x([0, \pi]) \rightarrow R$ as the controls, such that $u \in L^{2}(\Phi x([0, \pi]))$. It means that $t \rightarrow u(t)$ going from $J$ into $Y$ is measurable. Set $U(t):=\left\{u \in Y:\|u\|_{Y} \leq \vartheta\right\}$, where $\vartheta \in L^{2}\left(J, R^{+}\right)$. And restrict the admissible controls sets $\mathrm{U}_{\text {ad }}$ to be all $u \in L^{2}(\Phi x([0, \pi]))$ such that $\|u(\cdot, t)\|_{2} \leq \mathcal{V}(t)$, a.e. $t \in J$.

Denote that $x(t, y)=x(t)(y)$; then

$$
\begin{gathered}
f(t, x(t))(y)=e^{-t}+\frac{t^{1-\alpha}}{(t+6)^{2}} \sin (x(t))(y), \\
B(t) u(t)(y)=\left[\int_{0}^{1} q(\tau) u(\tau, t) d \tau\right](y) .
\end{gathered}
$$

It is easy to see that

$$
\|f(t, x(t))\|=e^{-t}+\frac{1}{36} t^{1-\alpha}\|x(t)\|:=\phi(t)+\rho t^{1-\alpha}\|x(t)\|,
$$

and, for any $x, y \in X$,

$$
\begin{aligned}
& \|f(t, x(t))-f(t, y(t))\| \\
& \quad \leq \frac{1}{(t+6)^{2}}\|x-y\|_{C_{1-\alpha}}:=\varphi(t)\|x-y\|_{C_{1-\alpha}} .
\end{aligned}
$$

Hence, all the conditions of Theorem 16 are satisfied, and the system (74) has an optimal pair solution.

\section{Conflict of Interests}

The authors declare that there is no conflict of interests regarding the publication of this paper.

\section{Acknowledgments}

This Project is supported by NNSF of China Grants nos. 11271087 and 61263006, Scientific Research Project of Guangxi Education Department, China (no. 2013YB074), Scientific Research Project of State Ethnic Affairs Commission of China (no. 12GXZ001), and the Innovation Project of Guangxi Graduate Education YCSZ2012062. 


\section{References}

[1] D. Baleanu, A. K. Golmankhaneh, and A. K. Golmankhaneh, "On electromagnetic field in fractional space," Nonlinear Analysis: Real World Applications, vol. 11, no. 1, pp. 288-292, 2010.

[2] A. Debbouche and M. M. El-Borai, "Weak almost periodic and optimal mild solutions of fractional evolution equations," Electronic Journal of Differential Equations, vol. 46, 8 pages, 2009.

[3] A. A. Kilbas, H. M. Srivastava, and J. J. Trujillo, "Theory and applications of fractional differential equations," in NorthHolland Mathematics Studies, vol. 204, Elservier Science B.V., Amsterdam, The Netherlands, 2006.

[4] V. Lakshmikantham and A. S. Vatsala, "Basic theory of fractional differential equations," Nonlinear Analysis: Theory, Methods \& Applications, vol. 69, pp. 2677-2682, 2008.

[5] Z. H. Liu and J. F. Han, "Integral boundary value problems for fractional order integrodifferential equations," Dynamic Systems \& Applications, vol. 21, pp. 535-548, 2012.

[6] Z. H. Liu and X. W. Li, "Existence and uniqueness of solutions for the nonlinear impulsive fractional differential equations," Communications in Nonlinear Science and Numerical Simulation, vol. 18, pp. 1362-1373, 2013.

[7] X. Y. Liu, Z. H. Liu, and X. Fu, "Relaxation in nonconvex optimal control problems described by fractional differential equations," Journal of Mathematical Analysis and Applications, vol. 409, no. 1, pp. 446-458, 2014.

[8] Z. H. Liu and X. W. Li, "On the controllability of impulsive fractional evolution inclusions in banach spaces," Journal of Optimization Theory and Applications, vol. 156, pp. 167-182, 2013.

[9] Z. H. Liu, J. H. Sun, and I. Szántó, "Monotone iterative technique for Riemann-Liouville fractional integro-differential equations with advanced arguments," Results in Mathematics, vol. 63, pp. 1277-1287, 2013.

[10] Z. H. Liu and J. H. Sun, "Nonlinear boundary value problems of fractional functional integro-differential equations," Computers and Mathematics with Applications, vol. 64, pp. 3228-3234, 2012.

[11] Z. H. Liu, X. W. Li, and J. H. Sun, "Controllability of nonlinear fractional impulsive evolution systems," Journal of Integral Equations and Applications, vol. 25, no. 3, pp. 395-405, 2013.

[12] K. X. Li and J. Peng, "Fractional resolvents and fractional evolution equations," Applied Mathematics Letters, vol. 25, no. 5, pp. 808-812, 2012.

[13] K. X. Li, J. G. Peng, and J. X. Jia, "Cauchy problems for fractional differential equations with Riemann-Liouville fractional derivatives," Journal of Functional Analysis, vol. 263, pp. 476510, 2012.

[14] M. P. Lazarevi'c and A. M. Spasi'c, "Finite-time stability analysis of fractional order timedelay systems: gronwall's approach," Mathematical and Computer Modelling, vol. 49, no. 3-4, pp. 475481, 2009.

[15] G. M. Mophou, "Optimal control of fractional diffusion equation," Computers and Mathematics with Applications, vol. 61, no. 1, pp. 68-78, 2011.

[16] I. Podlubny, Fractional Differential Equations, Academic Press, San Diego, Calif, USA, 1999.

[17] S. Zhang, "Monotone iterative method for initial value problem involving Riemann-Liouville fractional derivatives," Nonlinear Analysis, Theory, Methods and Applications, vol. 71, no. 5-6, pp. 2087-2093, 2009.
[18] N. Heymans and I. Podlubny, "Physical interpretation of initial conditions for fractional differential equations with RiemannLiouville fractional derivatives," Rheologica Acta, vol. 45, no. 5, pp. 765-771, 2006.

[19] M. M. El-Borai, "Some probability densities and fundamental solutions of fractional evolution equations," Chaos, Solitons and Fractals, vol. 14, no. 3, pp. 433-440, 2002.

[20] M. M. El-Borai, "The fundamental solutions for fractional evolution equations of parabolic type," Journal of Applied Mathematics and Stochastic Analysis, vol. 2004, no. 3, pp. 197211, 2004.

[21] J. R. Wang, Y. Zhou, and M. Medved, "On the solvability and optimal controls of fractional integrodifferential evolution systems with infinite delay," Journal of Optimization Theory and Applications, vol. 152, no. 1, pp. 31-50, 2012.

[22] J. R. Wang and Y. Zhou, "A class of fractional evolution equations and optimal controls," Nonlinear Analysis: Real World Applications, vol. 12, no. 1, pp. 262-272, 2011.

[23] Y. Zhou and F. Jiao, "Existence of mild solutions for fractional neutral evolution equations," Computers and Mathematics with Applications, vol. 59, no. 3, pp. 1063-1077, 2010.

[24] H. P. Ye, J. M. Gao, and Y. S. Ding, "A generalized Gronwall inequality and its application to a fractional differential equation," Journal of Mathematical Analysis and Applications, vol. 328, no. 2, pp. 1075-1081, 2007.

[25] S. Hu and N. S. Papageorgiou, Handbook of Multivalued Analysis (Theory), Kluwer Academic, Dordrecht, The Netherlands, 1997.

[26] A. Pazy, Semigroups of Linear Operators and Applications to Partial Differential Equations, Springer, New York, NY, USA, 1983.

[27] E. J. Balder, "Necessary and sufficient conditions for $L^{1}$-strongweak lower semicontinuity of integral functionals," Nonlinear Analysis, vol. 11, no. 12, pp. 1399-1404, 1987. 


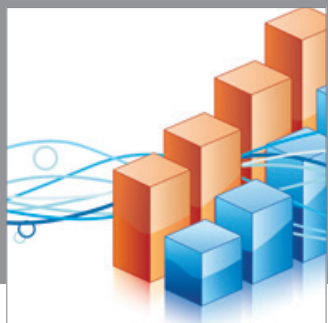

Advances in

Operations Research

mansans

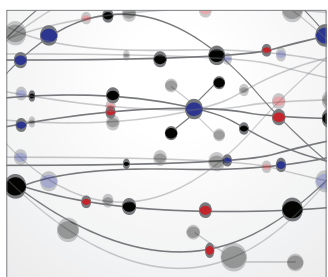

The Scientific World Journal
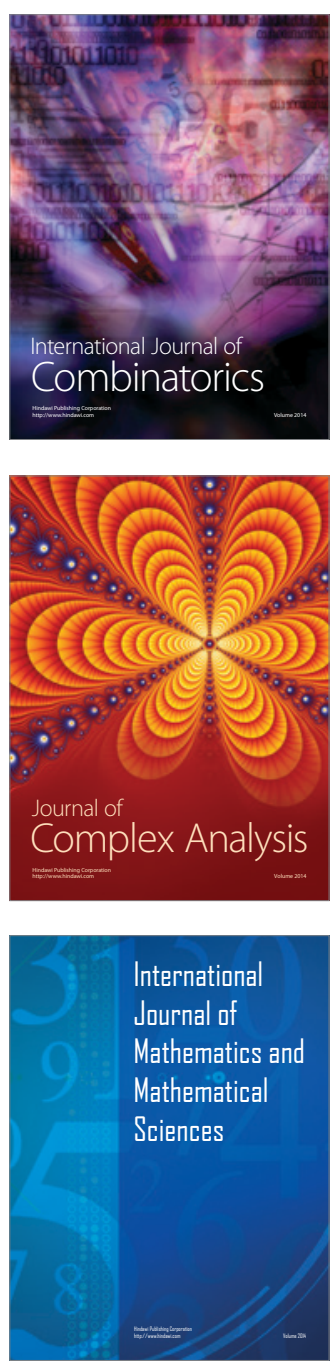
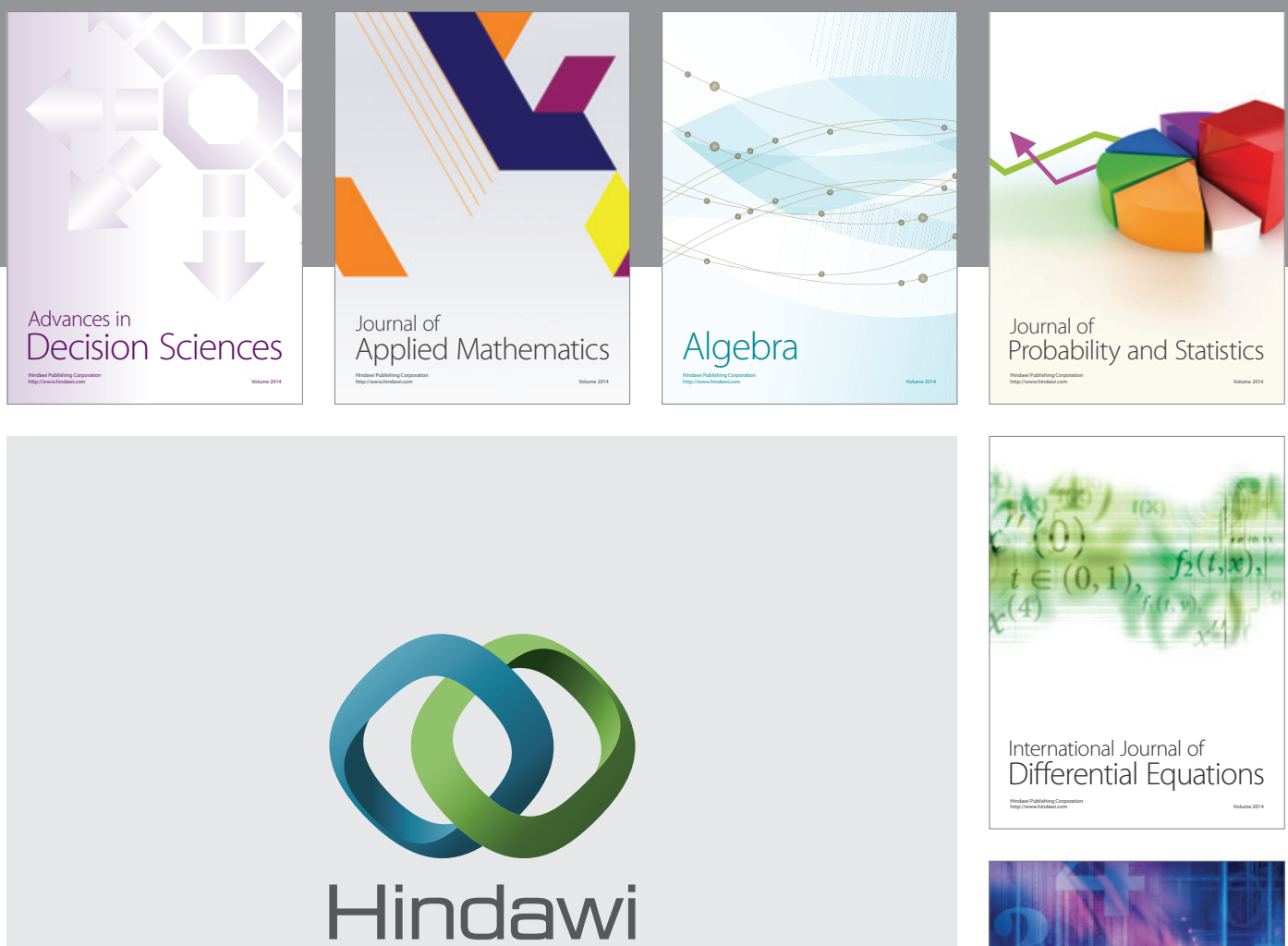

Submit your manuscripts at http://www.hindawi.com
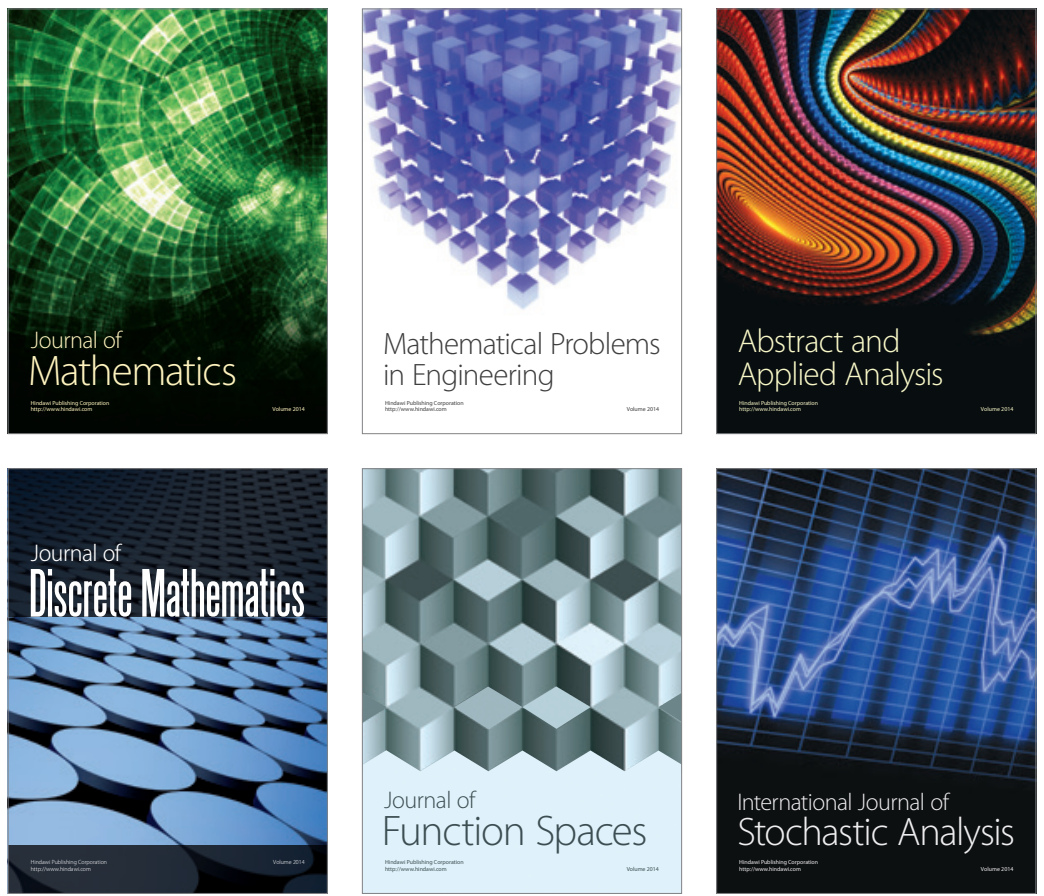

Journal of

Function Spaces

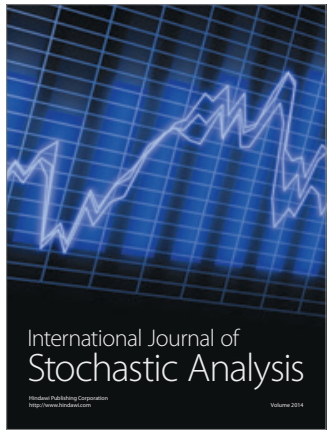

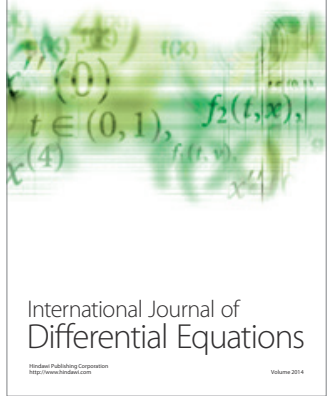
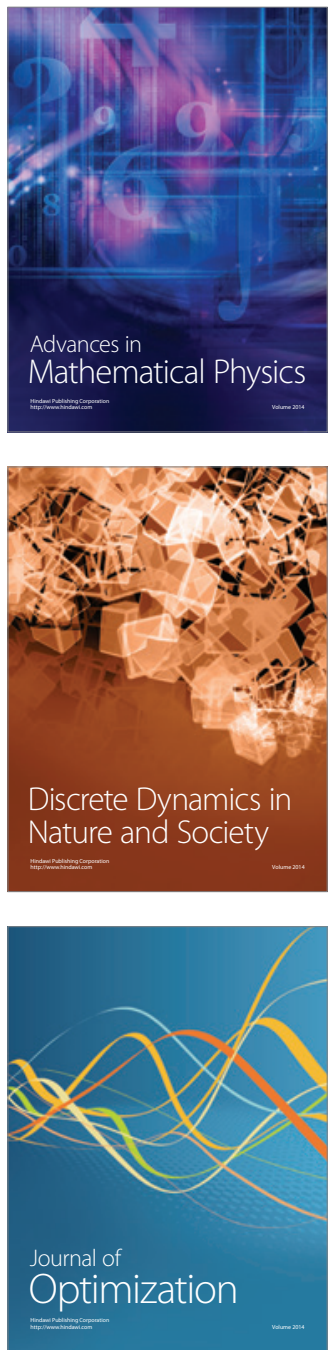